\title{
EDITORIAL
}

\section{Polysomnography for the management of progressive neuromuscular disorders}

\author{
F. Lofaso*,\#, M.A. Quera-Salva*
}

Invasive mechanical ventilation through a tracheostomy (TMV) is widely used to improve the survival of patients with severe restrictive respiratory disease caused by progressive neuromuscular disorders. For the last $20 \mathrm{yrs}$, there has been intense interest in noninvasive intermittent positive-pressure ventilation (NIMV) as a means of avoiding tracheostomy in these patients [1]. However, there is no evidence that NIMV is beneficial before the development of daytime hypercapnia, and NIMV may even be detrimental by causing a delay in seeking medical advice during acute exacerbations. In keeping with this possibility, a randomized study in which RAPHAËL et al. [2] evaluated the effect of NIMV on patients with Duchenne's muscular dystrophy before the stage of daytime hypercapnia, found an unexpected higher risk of death in the group receiving NIMV. It can be argued that this study did not use appropriate inclusion criteria for selecting the subset of patients most likely to benefit from "preventive" NIMV. Another limitation of this study is that effectiveness of NIMV during sleep (the most important period of NIMV) had not been evaluated at the time, whereas there is now firm evidence that leaks can occur when NIMV is delivered during sleep, to the possible detriment of NIMV efficacy [3, 4]. Furthermore, treatment compliance was assessed by patient interview and by home visits only, two subjective methods that failed to reliably estimate treatment compliance in a study of patients with sleep apnoea syndrome [5].

This issue of the journal contains an outstanding review by BOURKE and GIBSON [6] of the literature on interactions between sleep and breathing in neuromuscular patients and on the beneficial effects of NIMV during sleep. As pointed out by Bourke and GIBSON [6], although respiratory muscle function is generally more vulnerable during sleep (particularly during rapid eye movement) than during wakefulness, the criteria for using NIMV are mainly daytime parameters [7]. Furthermore, only recently have consensus guidelines incorporated criteria related to hypoventilation during sleep [8, 9], namely, symptoms suggesting hypercapnia $[8,9]$ during sleep and

\footnotetext{
*Physiologie-Explorations Fonctionnelles, Hôpital R. Poincaré, Garches, and ${ }^{\#}$ INSERM U 492, Hôpital H. Mondor, Créteil, France.

Correspondence: F. Lofaso, Physiologie-Explorations Fonctionnelles, Hôpital R. Poincaré, AP-HP, 92380 Garches, France. Fax: 11 147107943. E-mail: f.lofaso@rpc.ap-hop-paris.fr
}

oxygen desaturation for five consecutive minutes [9]. However, it has been reported that patients with neuromuscular disorders tend to underestimate symptoms such as fatigue before using mechanical ventilation and that this treatment induces a marked improvement $[10,11]$. In addition, as the oxyhaemoglobin dissociation curve has a sigmoid shape, pulse oximetry can be relatively insensitive for detecting a significant decrease in oxygen tension in arterial blood $\left(\mathrm{Pa}, \mathrm{O}_{2}\right)$ when $\mathrm{Pa}, \mathrm{O}_{2}$ values are above $55-60 \mathrm{mmHg}$; therefore, episodes of significant hypoventilation may be missed. Thus, if neuromuscular patients with nocturnal hypercapnia, even in the absence of daytime hypercapnia, are considered candidates for long-term ventilatory assistance, the indirect criteria of nocturnal hypoventilation proposed by the consensus guidelines [8,9] may be best viewed as sufficient but not indispensable for deciding to use NIMV.

This underlines the current disagreement between intensivists and traditional sleep specialists [12]. Clearly, caregivers involved in the medical management of neuromuscular patients should be encouraged to obtain sleep polysomnographies in these patients.

Which measurement techniques should be done during polysomnography? Probably those recently recommended for the diagnosis of sleep-related breathing disorders [13] with, in addition, transcutaneous partial pressure of carbon dioxide recording to detect a possible gradual rise in carbon dioxide tension in arterial blood during the various sleep stages.

When should polysomnography be performed? The present authors perform overnight polysomnography in all neuromuscular patients as early as possible as a baseline recording, and repeat the study according to the course of the neuromuscular disease to detect abnormalities during sleep and to demonstrate to the patient that treatment should be initiated. In addition, polysomnography should be repeated periodically after treatment initiation to evaluate the beneficial effects. It is clearly helpful to show the patients that the treatment corrects sleep and breathing abnormalities.

In addition, these follow-up polysomnography studies conducted during sleep can allow detection and correction of unexpected poor efficacy of treatment on ventilation and sleep stability, which is generally due either to asynchrony between the patient and the ventilator (this is particularly true in patients receiving NIMV, who are not yet entirely dependent 
on the ventilator) and/or to the occurrence of mouth leaks, which induce sleep fragmentation [3, 4]. The present authors found mouth leaks in $20 \%$ of patients during either NIMV or TMV with cuffless tubes [14].

Finally the review by Bourke and GiBson [6] gives us the opportunity to underline the need for performing polysomnography in neuromuscular patients both before and after treatment. Early polysomnography ensures detection of the earliest criteria for noninvasive intermittent positive-pressure ventilation, i.e., episodes of nocturnal hypercapnia and their adverse effects on sleep. Polysomnography is valuable for ensuring adequate sleep and ventilation during noninvasive intermittent positive-pressure ventilation and tracheostomy.

\section{References}

1. Rideau Y, Gatin G, Bach JR, Gines G. Prolongation of life in Duchenne's muscular dystrophy. Acta Neurol 1983; 5: 118-124.

2. Raphaël JC, Chevret S, Chastang C, Bouvet F. Randomised trial of preventive nasal ventilation in Duchenne muscular dystrophy. French Multicentre Cooperative Group on home mechanical ventilation assistance in Duchenne de Boulogne muscular dystrophy. Lancet 1994; 343: 1600-1604.

3. Meyer TJ, Pressman MR, Benditt J, et al. Air leaking through the mouth during nocturnal nasal ventilation: effect on sleep quality. Sleep 1997; 20: 561-519.

4. Teschler H, Stampa J, Ragette R, Konietzko N, Berthon-Jones M. Effect of mouth leak on effectiveness of nasal bilevel ventilatory assistance and sleep architecture. Eur Respir J 1999; 14: 1251-1257.
5. Rauscher H, Formaneck D, Popp W, Zwick H. Selfreported vs measured compliance with nasal CPAP for obstructive sleep apnea. Chest 1993; 103: 1675-1680.

6. Bourke SC, Gibson GJ. Sleep and breathing in neuromuscular disease. Eur Respir J 2002; 19: 1194-1201.

7. Raphaël J-C, Chevret S, Chastang C, Bouvet F. Home mechanical ventilation in Duchenne's muscular dystrophy: in search of therapeutic strategy. Eur Respir $J$ 1993; 3: 270-274.

8. Anonymous. Mechanical ventilation beyond the intensive care unit - report of consensus conference of the american college of chest physicians. Chest 1998; 113: 289s-344s.

9. Anonymous. Clinical indications for noninvasive positive pressure ventilation in chronic respiratory failure due to restrictive lung disease, COPD, and nocturnal hypoventilation - a consensus conference report. Chest 1999; 116: 521-534.

10. Smith PE, Calverley PM, Edwards RH, Evans GA, Campbell EJ. Practical problems in the respiratory care of patients with muscular dystrophy. $N$ Engl J Med 1987; 316: 1197-1205.

11. Raphaël J-C, Dazord A, Jaillard P, et al. Indices de satisfaction des patients atteints d'une dystrophie musculaire de Duchenne de Boulogne et ventilés à domicile. Rev Neurol 2002 (in press).

12. Rodenstein DO, Levy P. To sleep, perchance to leak. Eur Respir J 1999; 14: 1241-1243.

13. Anonymous. Sleep-related breathing disorders in adults: recommendations for syndrome definition and measurement techniques in clinical research. Sleep 1999; 22: 667-689.

14. Bach JR, Alba AS. Tracheostomy ventilation. A study of efficacy with deflated cuffs and cuffless tubes. Chest 1990; 97: 679-683. 